\title{
Photo-excitation of carotenoids causes cytotoxicity via singlet oxygen production
}

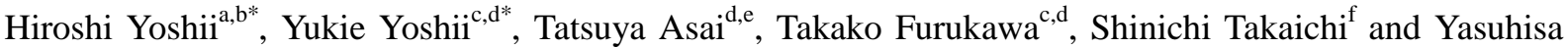
Fujibayashic $^{\mathrm{c}, \mathrm{d}}$

${ }^{a}$ Research Center for Radiation Emergency Medicine, National Institute of Radiological Science, Chiba,

Chiba 263-8555, Japan

${ }^{\mathrm{b}}$ Faculty of Medical Sciences, University of Fukui, Eiheiji, Fukui 910-1193, Japan

${ }^{c}$ Molecular Imaging Center, National Institute of Radiological Science, Chiba, Chiba 263-8555, Japan

dBiomedical Imaging Research Center, University of Fukui, Eiheiji, Fukui 910-1193, Japan

${ }^{\mathrm{e}}$ Faculty of Engineering, University of Fukui, Fukui, Fukui 910-8507, Japan

${ }^{\mathrm{f}}$ Department of Biology, Nippon Medical School, Kawasaki, Kanagawa 211-0063, Japan

"Corresponding authors. Addresses: Research Center for Radiation Emergency Medicine (H. Yoshii) and Molecular Imaging Center (Y. Yoshii), National Institute of Radiological Science, 4-9-1 Anagawa, Inage-ku, Chiba, Chiba 263-8555, Japan. Fax: +81 432841769 (H. Yoshii), +81 432060818 (Y. Yoshii). E-mail addresses: yoshii@ nirs.go.jp (H. Yoshii), yukiey@nirs.go.jp (Y. Yoshii).

(C) 2011. This manuscript version is made available under the Elsevier user license http://www.elsevier.com/open-access/userlicense/1.0/ 


\section{Abstract}

Carotenoids, natural pigments widely distributed in algae and plants, have a conjugated double bond system. Their excitation energies are correlated with conjugation length. We hypothesized that carotenoids whose energy states are above the singlet excited state of oxygen (singlet oxygen) would possess photosensitizing properties. Here, we demonstrated that human skin melanoma (A375) cells are damaged through the photo-excitation of several carotenoids (neoxanthin, fucoxanthin and siphonaxanthin). In contrast, photo-excitation of carotenoids that possess energy states below that of singlet oxygen, such as $\beta$-carotene, lutein, loroxanthin and violaxanthin, did not enhance cell death. Production of reactive oxygen species (ROS) by photo-excited fucoxanthin or neoxanthin was confirmed using a reporter assay for ROS production with HeLa Hyper cells, which express a fluorescent indicator protein for intracellular ROS. Fucoxanthin and neoxanthin also showed high cellular penetration and retention. Electron spin resonance spectra using 2,2,6,6-tetramethil-4-piperidone as a singlet oxygen trapping agent demonstrated that singlet oxygen was produced via energy transfer from photo-excited fucoxanthin to oxygen molecules. These results suggest that carotenoids such as fucoxanthin, which are capable of singlet oxygen production through photo-excitation and show good penetration and retention in target cells, are useful as photosensitizers in photodynamic therapy for skin disease.

Keywords: carotenoid, reactive oxygen species, singlet oxygen, electron spin resonance 


\section{Introduction}

Carotenoids play two essential roles in the photosynthetic system in plants; light harvesting and photoprotection [1]. Light harvesting refers to the collection of sunlight and transfer of excitation energy to chlorophylls. Through energy transfer, the ground $\left(\mathrm{S}_{0}\right)$ state of chlorophylls is excited to the $\mathrm{Q}_{\mathrm{y}}\left(\mathrm{S}_{1}\right)$ or $\mathrm{Q}_{\mathrm{x}}\left(\mathrm{S}_{2}\right)$ states, and this excitation energy is delivered to the reaction center of the photosynthetic system. Photoprotection refers to the prevention of cell damage induced by oxygen molecules in the excited ${ }^{1} \Delta_{\mathrm{g}}$ state (singlet oxygen: ${ }^{1} \mathrm{O}_{2}$ ), which are usually produced by the photosensitization of over-excited chlorophylls and have very high toxicity among reactive oxygen species (ROS). Photoprotective carotenoids take the excitation energy away from excited chlorophylls and convert this energy to heat (non-radiative energy dissipation). Quenching of produced ${ }^{1} \mathrm{O}_{2}$ via energy transfer from ${ }^{1} \mathrm{O}_{2}$ to carotenoids is also an important photoprotective role of carotenoids.

These two conflicting roles, light harvesting and photoprotection, are the result of a variety of energy levels among the different carotenoids. Energies of the first and second singlet excited states, the $S_{1}$ and $S_{2}$ states, are inversely correlated with conjugation length [2]. Note that the energy of the triplet first excited state, the $\mathrm{T}_{1}$ state, of carotenoids is estimated to be half of the $S_{1}$ state energy $[3,4]$; thus, the energy of the $T_{1}$ state is also inversely correlated with conjugation length. Fucoxanthin (7OA), neoxanthin (8A), siphonaxanthin (8O), lutein (9 $\beta 1)$ and loroxanthin (9 $\beta 1)$ are typical light-harvesting carotenoids [5-9]. Here, the notation for conjugation length is based on Polívka and Sundström [10]: number, number of 
conjugated $\mathrm{C}=\mathrm{C}$ bonds; $\mathrm{O}$, carbonyl group; $\mathrm{A}$, allene group; and $\beta n$, number of conjugated $\beta$-end groups. The $S_{2}$ states of these carotenoids are located above that of chlorophylls. Carotenoids known to act as photoprotective carotenoids have lower $S_{1}$ states than chlorophylls or lower $\mathrm{T}_{1}$ states than ${ }^{1} \mathrm{O}_{2}$. In contrast, lycopene (11), $\beta$-carotene (9ß2), lutein

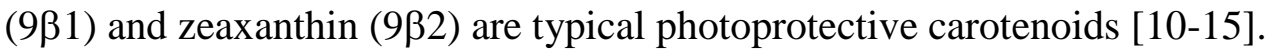

Light-harvesting carotenoids generally have shorter conjugation length than photoprotective carotenoids. Lutein can act both as a light-harvesting carotenoid and a photoprotective carotenoid, as lutein has a higher energy $S_{2}$ state than chlorophylls, but the $S_{1}$ state is slightly lower than chlorophylls, while the $\mathrm{T}_{1}$ state is lower than ${ }^{1} \mathrm{O}_{2}$. Thus, the energy transfer between chlorophylls and carotenoids, and that from ${ }^{1} \mathrm{O}_{2}$ to carotenoids have been thoroughly studied [5-15]. However, energy transfer from excited carotenoids to oxygen molecules has not been confirmed to date in carotenoids, which have the higher $T_{1}$ states than ${ }^{1} \mathrm{O}_{2}$.

On the other hand, Xia et al. [16] detected ${ }^{1} \mathrm{O}_{2}$ production after UVA irradiation of retinyl palmitate (RP), which is a retinyl ester commonly used as sunscreen. Although it is not a carotenoid, it has a similar, but shorter, conjugated double bond system $(n=4)$. Therefore, excitation energy of the $S_{0}-S_{2}$ transition of RP is in the UVA region. Xia et al. [16] showed that UVA-irradiated RP produces ${ }^{1} \mathrm{O}_{2}$ on electron spin resonance (ESR) spectra. As RP has a conjugation length of $n=4$, the $\mathrm{T}_{1}$ state energy of RP is higher than that of ${ }^{1} \mathrm{O}_{2}$ and ${ }^{1} \mathrm{O}_{2}$ can be produced via energy transfer from excited RP to oxygen molecules. Here, we hypothesized 
that some photo-excited carotenoids, which have the $\mathrm{T}_{1}$ states above ${ }^{1} \mathrm{O}_{2}$, can produce ${ }^{1} \mathrm{O}_{2}$ in a similar manner as UVA-irradiated RP.

In the present study, we investigated whether some photo-excited carotenoids have photosensitizing ability and whether they are able to produce ROS. We also examined ${ }^{1} \mathrm{O}_{2}$ production using an ESR spin trapping technique with a singlet oxygen trapping agent, 2,2,6,6-tetramethil-4-piperidone (TMPD) (Nacalai Tesque, Kyoto, Japan) [17].

\section{Materials and Methods}

\section{Preparation of carotenoids}

For extraction of pigments, spinach leaves, green algae (Caulerpa lentillifera, Cladophora sp.) and brown algae (Undaria pinnatifida) were washed with $10 \mathrm{mM}$ HEPES buffer (pH 7.4) and homogenized in $100 \%$ acetone on ice in the dark. Pigment extracts were centrifuged and supernatants were filtered (0.2 $\mu \mathrm{m}$ LCR13-LG; Millipore, Bedford, MA). Extracted pigments were separated by silica gel TLC (Silicagel 60, 111845; Merck, Darmstadt, Germany), using a mixture of hexane and acetone (7:3) as a development solvent. Further purification was performed using an HPLC system (Shimadzu, Kyoto, Japan) with a Wakosil 5C18 column $(4.6 \times 150 \mathrm{~mm}$; Wako Pure Chemical Industries, Osaka, Japan) and methanol as a mobile phase. Purity of carotenoids was analyzed using an HPLC system with a Partisil-5 ODS-3 column $(4 \times 250 \mathrm{~mm}$; GL Science, Tokyo, Japan $)$ and elution conditions using ion-pairing solution, as described previously [18], and >99\% pure carotenoids were then used in this 
study. Seven carotenoids were prepared: $\beta$-carotene, lutein, violaxanthin and 9'-cis neoxanthin from spinach leaves; loroxanthin from Cladophora sp.; siphonaxanthin from Caulerpa lentillifera; fucoxanthin from Undaria pinnatifida. Table 1 lists the conjugation length, which follows a previously reported notation style [10], energy of the $S_{1}$ state $[19,20]$, energy of the $T_{1}$ state (estimated as half of the $S_{1}$ state energy), and the energy difference between the ${ }^{1} \mathrm{O}_{2}$ and $\mathrm{T}_{1}$ state of carotenoids examined in this study $(\Delta E)$.

\section{Light source}

Light emitting diode (LEDs) (BL503B2CA1A01; Linkman, Fukui, Japan), were used for blue light (BL) irradiation in this study. For irradiation of 96-well cell culture plates, LEDs were mounted with equal spaces to match up with the wells, and cells in each well were irradiated with BL from one LED from the bottom of the plate. The irradiation system for a flat ESR sample vessel ES-LC12 (JEOL, Tokyo, Japan) contained two facing banks of LEDs (each bank had 7 LEDs), and samples in the vessel were irradiated equally with BL from both sides. A regulated DC power supply was used to provide $3.6 \mathrm{~V}$ to the LEDs connected in parallel. The electric current for each LED was $20 \mathrm{~mA}$, and the expected luminous intensity was $3000 \mathrm{mcd}$. The directly observed photon count was $200 \mu \mathrm{mol}$ photons $\cdot \mathrm{m}^{-2} \cdot \mathrm{s}^{-1}$. The wavelength emitted by the LEDs was $468 \mathrm{~nm}$, and the full width at half maximum wavelength was $25 \mathrm{~nm}$. BL supplied by LEDs was able to effectively excite carotenoids from the ground state to the optically allowed $\mathrm{S}_{2}$ state. 


\section{Effects of photo-excited carotenoids on human melanoma cells}

Human melanoma cells (A375; American Type Culture Collection, CRL-1619) were used in this study. Cells were incubated in a humidified atmosphere of $5 \% \mathrm{CO}_{2}$ in air at $37^{\circ} \mathrm{C}$. Dulbecco's modified Eagle's medium (DMEM 11995-065; Invitrogen, Carlsbad, CA) supplemented with $10 \%$ fetal bovine serum and antibiotics was used for cell growth. Exponentially growing cells were used in all experiments.

For assay, cells were plated in 96-well plates (BD Falcon, Franklin Lakes, NJ) at a density of $5 \times 10^{3}$ cells in $100 \mu \mathrm{l}$ of growth medium per well, and were preincubated for $24 \mathrm{~h}$. The medium was changed to growth medium supplemented with $2 \mu \mathrm{M}$ carotenoids (Car); $\beta$-carotene $(\beta)$, lutein (Lut), loroxanthin (Loro), violaxanthin (Viola), neoxanthin (Neo), fucoxanthin (Fuco) and siphonaxanthin (Sx). Carotenoids were dissolved in tetrahydrofuran (THF) and then added to growth medium. The final concentration of THF in medium was $0.5 \%$. Control culture received only THF (vehicle alone). After an additional $5 \mathrm{~h}$ of incubation with or without BL irradiation, the number of viable cells was calculated by the trypan blue dye-exclusion method (15250-061; Invitrogen). Thus, cell treatments were classified into the following groups: (Car-, BL-); (Car-, BL+); (Car+, BL-); and (Car+, BL+). Note that latter groups contained all carotenoids; $\beta$, Lut, Loro, Viola, Neo, Fuco and Sx.

\section{ROS production by photo-excited carotenoids}


HeLa Hyper cells (Evrogen JSC, Moscow, Russia), which express a fluorescent indicator protein for intracellular ROS [21], were plated in 96-well plates at a density of $5 \times$ $10^{3}$ cells in $100 \mu \mathrm{l}$ of growth medium per well, and were preincubated for $24 \mathrm{~h}$. Medium was then replaced with medium alone or medium supplemented with $2 \mu \mathrm{M}$ carotenoids (Car) shown to damage cells by photo-excitation; neoxanthin (Neo) or fucoxanthin (Fuco). After $3 \mathrm{~h}$ of incubation, an additional $2 \mathrm{~h}$ of incubation with or without BL irradiation was performed. Before BL irradiation, medium for some samples was replaced with fresh growth medium without carotenoids in order to eliminate carotenoids from the medium (medium change, MC). Treatments were, therefore, classified into the following eight groups: A (Car-, BL-, MC-); B (Car-, BL+, MC-); C (Neo +, BL-, MC-); D (Fuco+, BL-, MC-); E (Neo+, BL+, MC-); F (Fuco+, BL+, MC-); G (Neo+, BL+, MC+); and H (Fuco+, BL+, MC+). After incubation, fluorescent images were obtained using a fluorescence microscope (IX70; Olympus, Tokyo, Japan).

\section{ESR measurements}

In this study, we examined whether photo-excited fucoxanthin, whose $\mathrm{T}_{1}$ state energy is higher than that of ${ }^{1} \mathrm{O}_{2}$ (Table 1 ), is able to produce ${ }^{1} \mathrm{O}_{2}$ via energy transfer to oxygen molecules, using ESR measurements with TMPD as a ${ }^{1} \mathrm{O}_{2}$ trapping agent. ESR spectra were measured with an ESR spectrometer (JES-RE3XR; JEOL, Tokyo, Japan) under the following conditions: magnetic field, $335.9 \pm 7.9 \mathrm{mT}$; microwave power, $10 \mathrm{~mW}$; microwave frequency, 
9.43 GHz; modulation width, $0.079 \mathrm{mT}$; modulation frequency, $100 \mathrm{kHz}$; time constant, $0.3 \mathrm{~s}$; and sweep time, $4.0 \mathrm{~min}$. The sample contained $5 \mathrm{mM}$ fucoxanthin in ethanol and $100 \mathrm{mM}$ TMPD in water $(16: 1, \mathrm{v} / \mathrm{v})$. The sample solution was set to a flat ESR sample vessel, and this was irradiated with BL using the above-described irradiation system for ESR measurement for 0, 20, 40, 60, 80 or 100 min. ESR measurements started immediately after irradiation. The following samples were also prepared: a sample containing fucoxanthin and TMPD treated under hypoxic conditions with Anaero pack (A-07; Mitsubishi Gas Chemical Company, Tokyo, Japan) for 6 hours; a sample containing $1 \mathrm{mM}$ histidine, a widely used ${ }^{1} \mathrm{O}_{2}$ quencher [22], as well as fucoxanthin and TMPD for a ${ }^{1} \mathrm{O}_{2}$ quenching study; and a sample containing TMPD and $\beta$-carotene instead of fucoxanthin. Irradiation and ESR measurements for these samples were performed in a similar manner as described above. Samples containing only fucoxanthin or TMPD were used as controls. Controls were irradiated with BL for 60 min before ESR measurements.

\section{Statistical analysis}

Data are expressed as means and standard deviation. $P$ values were calculated by ANOVA for comparison between multiple groups. $P$ values of $<0.05$ were considered to be statistically significant.

\section{Results}




\section{Cytotoxicity induced by photo-excitation of carotenoids in human melanoma cells}

Figure 1 shows the viability of A375 cells treated with or without carotenoids and BL irradiation relative to untreated controls. The viability of cells treated with fucoxanthin or neoxanthin under BL irradiation was less than $20 \%$ when compared to untreated controls $(\mathrm{P}<0.05)$, while cells without BL irradiation showed no significant decrease in viability. Although the viability of cells treated with siphonaxanthin and BL irradiation was also less than $20 \%$ that of untreated controls $(\mathrm{P}<0.05)$, cell viability decreased to approximately $60 \%$ without BL irradiation. For cells treated with lutein, loroxanthin and violaxanthin, viability was $20 \%-50 \%$ lower both with and without BL irradiation, as compared with controls $(\mathrm{P}<0.05)$. There were no significant differences between the viability of cells treated with $\beta$-carotene with or without BL irradiation and that for untreated cells. The viability of cells treated only with BL irradiation decreased slightly $(\mathrm{P}<0.05)$.

\section{ROS production by photo-excited carotenoids}

As fucoxanthin or neoxanthin treatment with BL irradiation showed apparent cytotoxicity (Fig. 1), we next examined ROS production by photo-excited fucoxanthin or neoxanthin with HeLa Hyper cells by fluorescence microscopy (Fig. 2). Substantial fluorescence signals were detected in groups $\mathrm{E}(\mathrm{Neo}+, \mathrm{BL}+, \mathrm{MC}-)$ and $\mathrm{F}$ (Fuco+, BL+, MC-), while groups A (Car-, BL-, MC-), B (Car-, BL+, MC-), C (Neo+, BL-, MC-) and D (Fuco+, BL-, MC-) showed negligible signals. This means that ROS are produced by photo-excited 
fucoxanthin or neoxanthin. Moreover, groups $\mathrm{G}(\mathrm{Neo}+, \mathrm{BL}+, \mathrm{MC}+)$ and $\mathrm{H}(\mathrm{Fuco}+, \mathrm{BL}+$, $\mathrm{MC}+$ ) also showed substantial fluorescence signals, similarly to groups $\mathrm{E}$ and F. Thus, ROS signals were detected regardless of medium change. This suggests that neoxanthin and fucoxanthin possess high cellular penetration and retention abilities.

\section{ESR measurements}

In order to examine whether carotenoid photo-excitation induces production of ${ }^{1} \mathrm{O}_{2}$,

ESR spectra measurements were performed with a representative carotenoid, fucoxanthin.

Measured ESR spectra were normalized against peak areas for Mn markers (Fig. 3). Three equivalent-height peaks between Mn markers indicated oxidization of TMPD by ${ }^{1} \mathrm{O}_{2}$. As TMPD is able to selectively trap ${ }^{1} \mathrm{O}_{2}[17],{ }^{1} \mathrm{O}_{2}$ production can be assessed based on the peak area of oxidized TMPD. Figure 4 shows the correlation between BL irradiation time and ${ }^{1} \mathrm{O}_{2}$ production, as measured by TMPD peak area. For samples containing fucoxanthin and TMPD, production of oxidized TMPD was progressively enhanced with photo-irradiation time from 0 to $60 \mathrm{~min}$, reaching a plateau at $60 \mathrm{~min}$. Inhibition with histidine against ${ }^{1} \mathrm{O}_{2}$ production led to a decrease in oxidized TMPD signals. There were almost no peaks in ESR spectra for the sample containing fucoxanthin and TMPD with hypoxic treatment. For the sample containing $\beta$-carotene and TMPD, ESR spectra showed no peaks. The ESR spectra for control samples, containing only fucoxanthin or TMPD, also showed no peaks (data not shown). 


\section{Discussion}

In the present study, we found that photo-excited fucoxanthin or neoxanthin induces the death of human melanoma cells. These carotenoids produce ROS through photo-excitation; thus, fucoxanthin and neoxanthin have good photosensitizing ability. We also detected ${ }^{1} \mathrm{O}_{2}$ production via energy transfer from photo-excited fucoxanthin to oxygen molecules.

Kotake-Nara et al. [23] examined the effects of carotenoid supplementation in human prostate cancer cells (PC-3, DU145 and LNCaP cell lines). They incubated cells for $72 \mathrm{~h}$ in medium containing 15 carotenoids at concentrations of 5,10 or $20 \mu \mathrm{M}$. They demonstrated that supplementation with carotenoids, including fucoxanthin and neoxanthin, had concentration-dependent antiproliferative effects, and that low concentrations $(5 \mu \mathrm{M})$ of fucoxanthin or neoxanthin had no adverse effects on cell viability. On the other hand, we demonstrated that, with BL irradiation, treatment with low concentrations $(2 \mu \mathrm{M})$ of fucoxanthin or neoxanthin can damage human melanoma cells. This indicates that photo-excitation enhances the cell damage caused by supplementation with low-dose fucoxanthin or neoxanthin.

Kotake-Nara et al. [24] also noted that the antiproliferative effects of supplementation with fucoxanthin or neoxanthin at $20 \mu \mathrm{M}$ is caused by apoptosis though caspase- 3 activation, rather than by ROS production. In this study, we found that non-excited fucoxanthin or neoxanthin did not produce ROS (Fig. 4C or D) and this is consistent with the 
results of Kotake-Nara et al. [24]. On the other hand, we found that photo-excited fucoxanthin or neoxanthin produce ROS (Fig. 2E or F), and the cell death induced by photo-excited fucoxanthin or neoxanthin would probably be caused primarily by ROS production.

The ESR spectra for photo-excited fucoxanthin showed time-dependent increases in the signal intensity of oxidized TMPD, indicating ${ }^{1} \mathrm{O}_{2}$ production (Fig. 4), while inhibition of ESR signals by histidine, a ${ }^{1} \mathrm{O}_{2}$ quencher, was confirmed (Fig. 4). These results indicate that photo-excited fucoxanthin can produce ${ }^{1} \mathrm{O}_{2}$. With hypoxic treatment, no ${ }^{1} \mathrm{O}_{2}$ peak was observed in the ESR spectra, even when the sample containing fucoxanthin and TMPD was irradiated with $\mathrm{BL}$ (Fig. 4). This indicates that oxygen is necessary for ${ }^{1} \mathrm{O}_{2}$ production by photo-excited fucoxanthin. Therefore, ${ }^{1} \mathrm{O}_{2}$ must be produced via energy transfer from photo-excited fucoxanthin to oxygen molecules. Considering that the ground state of oxygen is triplet $\left({ }^{3} \Sigma_{\mathrm{g}}^{-}\right)$and triplet-to-triplet energy transfer should occur in energy transfer between molecules according to the spin symmetry, the energy transfer would occur from the $\mathrm{T}_{1}$ state of fucoxanthin to the ground state of the oxygen molecule.

There have been no previous studies on energy transfer from the $T_{1}$ state of carotenoids to the ground state of oxygen molecules. However, this type of energy transfer can occur in fucoxanthin, as the energy of the $\mathrm{T}_{1}$ state of fucoxanthin is higher than that of ${ }^{1} \mathrm{O}_{2}$ (Table 1). In fact, the photo-excitation of $\beta$-carotene, which has a much lower $T_{1}$ state than ${ }^{1} \mathrm{O}_{2}$ (Table 1), cannot produce ${ }^{1} \mathrm{O}_{2}$ (Fig. 4). It is therefore reasonable to suggest that photo-excited fucoxanthin is able to produce ${ }^{1} \mathrm{O}_{2}$, a toxic ROS, via energy transfer to oxygen 
molecules around the cells, and this would induce damage in the cells. Neoxanthin, whose $\mathrm{T}_{1}$ state is slightly lower than the ${ }^{1} \mathrm{O}_{2}$ state (Table 1), is also able to produce ROS via photo-excitation (Fig. 2E). As the energy levels in molecules are typically spread in solution, the energy of neoxanthin in the $\mathrm{T}_{1}$ state can probably be transferred to oxygen molecules, and because the $S_{2}$ state of fucoxanthin or neoxanthin results from the ground state after BL irradiation, the $T_{1}$ state must be produced from the $S_{2}$ state. Further study is necessary in order to elucidate the pathways responsible for the $T_{1}$ state in fucoxanthin and neoxanthin. Irrespective, this is the first observation of ${ }^{1} \mathrm{O}_{2}$ production via energy transfer from excited carotenoids to oxygen molecules.

The present results also showed that fucoxanthin exhibits good cellular penetration and retention (Fig. $2 \mathrm{H}$ ), in addition to a good capacity for ${ }^{1} \mathrm{O}_{2}$ production via photo-excitation (Fig. 4). These features of fucoxanthin would be useful for medical applications such as photodynamic therapy (PDT) for skin diseases [25]. In PDT, ${ }^{1} \mathrm{O}_{2}$ produced by energy transfer from photo-excited photosensitizers to oxygen molecules attacks target cells. For skin diseases, 5-aminolevulinic acid (ALA), which is endogenously converted to protoporphyrin IX (PpIX), an efficient photosensitizer in response to BL, is applied as a photosensitizing agent to the affected part of the skin and BL irradiation is then performed [26]. However, ALA must be applied to the affected site in large amounts because of its low membrane permeability and loss in metabolism [26]. As noted in the present study, fucoxanthin shows high penetration and retention, and is able to produce ${ }^{1} \mathrm{O}_{2}$ through photo-excitation, which 
damages cells even at low concentrations. Thus, fucoxanthin is a potentially useful photosensitizer in PDT for skin diseases, and may be able to overcome the disadvantages of associated with ALA.

\section{Acknowledgements}

We would like to thank H. Takagi (Life Science Research Laboratory, University of Fukui, Japan) for technical assistance and members of the Biomedical Imaging Research Center of University of Fukui, Japan, for helpful discussion. Part of this study was supported by Research for Promoting Technological Seeds from the Japan Science and Technology Agency. 


\section{References}

[1] P. Horton, A. Ruban, Molecular design of the photosystem II light-harvesting antenna: photosynthesis and photoprotection, J. Exp. Bot. 56 (2005) 365-373.

[2] H.A. Frank, R. Farhoosh, R. Gebhard, J. Lugtenburg, D. Gosztola, M.R. Wasielewski, The dynamics of the S1 excited states of carotenoids, Cham. Phys. Lett. 207 (1993) 88-92.

[3] P. Tavan, K. Schulten, Electronic excitations in finite and infinite polyenes, Phys. Rev. B 36 (1987) 4337-4358.

[4] C.C. Gradinaru, J.T.M. Kennis, E. Papagiannakis, I.H.M. van Stokkum, R.J. Cogdell, G.R. Fleming, R.A. Niederman, R. van Grondelle, An unusual pathway of excitation energy deactivation in carotenoids: singlet-to-triplet conversion on an ultrafast timescale in a photosynthetic antenna, Proc. Natl. Acad. Sci. USA 98 (2001) 2364-2369.

[5] N. Gildenhoff, S. Amarie, K. Gundermann, A. Beer, C. Büchel, J. Wachtveitl, Oligomerization and pigmentation dependent excitation energy transfer in fucoxanthin-chlorophyll proteins, Biochim. Biophys. Acta 1797 (2009) 543-549.

[6] R. Croce, M. G. Müller, R. Bassi, A. R. Holzwarth, Carotenoid-to-chlorophyll energy transfer in recombinant major light-harvesting complex (LHCII) of higher plants. I.

Femtosecond transient absorption measurements, Biophys. J. 80 (2001) 901-915.

[7] Y. Yoshii, S. Takaichi, T. Maoka, T. Inoue, Photosynthetic pigment composition in the primitive green alga Mesostigma viride (prasinophyceae): phylogenetic and evolutionary implications, J. Phycol. 39 (2003) 570-576.

[8] Y. Yoshii, Diversity and evolution of photosynthetic antenna system in green plants, Phycol. Res. 54 (2006) 220-229.

[9] J. L. Garrido, F. Rodriguez, M. Zapata, Occurrence of loroxanthin, loroxanthin decenoate, and loroxanthin dodecenoate in Tetraselmis species (prasinophyceae, chlorophyta), J. Phycol. 45 (2009) 366-374.

[10] T. Polívka, V. Sundström, Ultrafast dynamics of carotenoid excited states-From solution to natural and artificial systems, Chem. Rev. 104 (2004) 2021-2071.

[11] P. Di Mascio, S. Kaiser, H. Sies, Lycopene as the most efficient biological carotenoid singlet oxygen quencher, Arch. Biochem. Biophys. 274 (1989) 532-538.

[12] W.G. Siems, O. Sommerburg, F.J.G.M. van Kuijk, Lycopene and $\beta$-carotene decompose more rapidly than lutein and zeaxanthin upon exposure to various pro-oxidants in vitro, BioFactors 10 (1999) 105-113.

[13] F.Q. Schafer, H.P. Wang, E.E. Kelley, K.L. Cueno, S.M. Martin, G.R. Buettner, Comparing $\beta$-carotene, vitamin $\mathrm{E}$ and nitric oxide as membrane antioxidants, Biol. Chem. 383 (2002) 671-681. 
[14] A.V. Ruban, R. Berera, C. Ilioaia, I.H.M. van Stokkum, J.T.M. Kennis, A.A. Pascal, H. van Amerongen, B. Robert, P. Horton, R. van Grondelle, Identification of a mechanism of photoprotective energy dissipation in higher plants, Nature 450 (2007) 575-579.

[15] B. Demming-Adams, A.M. Gilmore, W.W. Adams III, In vivo functions of carotenoids in higher plants, FASEB J. 10 (1996) 403-412.

[16] Q. Xia, J.J. Yin, S.-H. Cherng, W.G. Wamer, M. Boudreau, P.C. Howard, P.P. Fu, UVA photoirradiation of retinyl palmitate-Formation of singlet oxygen and superoxide, and their role in induction of lipid peroxidation, Toxicol. Lett. 163 (2006) 30-43.

[17] Y. Tokuoka, A. Niitsu, N. Watabe, T.N. Murakami, N. Kawashima, ESR spectroscopy of singlet oxygen generated by protoporphyrin IX in aqueous surfactant solutions, J. Oleo Sci. 52 (2003) 135-140.

[18] Y. Yoshii, S. Takaichi, T. Maoka, S. Hanada, I. Inoue, Characterization of two unique carotenoid fatty acid esters from Pterosperma cristatum (prasinophyceae, chlorophyta), J. Phycol. 38 (2002) 297-303.

[19] D.M. Niedzwiedzki, M.M. Enriquez, A.M. LaFountain, H.A. Frank, Ultrafast time-resolved absorption spectroscopy of geometric isomers of xanthophylls, Chem. Phys. 373 (2010) 80-89.

[20] D. Zigmantas, R. G. Hiller, F.P. Sharples, H.A. Frank, V. Sundström, T. Polívka, Effect of a carotenoid carbonyl group on the photophysical properties of carotenoids, Phys. Chem. Chem. Phys. 6 (2004) 3009-3016

[21] K.N. Markvicheva, E.A. Bogdanova, D.B. Staroverov, S. Lukyanov, V.V. Belousov, Imaging of intracellular hydrogen peroxide production with HyPer upon stimulation of HeLa cells with epidermal growth factor, Methods Mol. Biol. 476 (2009) 76-83.

[22] X. Zhai, X. Zhou, M. Ashraf, Interaction of singlet oxygen with 5'-nucleotidase in rat hearts, J. Mol. Cell Cardiol. 27 (1995) 2453-2464.

[23] E. Kotake-Nara, M. Kushiro, H. Zhang, T. Sugawara, K. Miyashita, A. Nagao, Carotenoids affect proliferation of human prostate cancer cells, J. Nutr. 131 (2001) 3303-3306.

[24] E. Kotake-Nara, A. Asai, A. Nagao, Neoxanthin and fucoxanthin induce apoptosis in PC-3 human prostate cancer cells, Cancer Lett. 220 (2005) 75-84.

[25] J.P. Celli, B.Q. Spring, I. Rizvi, C.L. Evans, K.S. Samkoe, S. Verma, B.W. Pogue, T. Hasan, Imaging and photodynamic therapy: mechanisms, monitoring, and optimization, Chem. Rev. 110 (2010) 2795-2838.

[26] M.A. MacCormack, Photodynamic therapy in dermatology: an update on applications and outcomes, Semin. Cutan. Med. Surg. 27 (2008) 52-62. 


\section{Figure legends}

Figure 1. Viability of A375 cells treated with or without carotenoids and BL irradiation.

Viable cells were counted by microscopy. Carotenoids used were $\beta$-carotene ( $\beta$ ), lutein (Lut), loroxanthin (Lo), violaxanthin (Viola), neoxanthin (Neo), fucoxanthin (Fuco) and siphonaxanthin (Sx). Control cells were not treated with carotenoids or BL (Cont. BL-), and examined cells were either irradiated or not irradiated with BL (BL+ or BL-).

Figure 2. ROS production by photo-excited fucoxanthin or neoxanthin in Hela Hyper cells. Images show fluorescence microscopy of HeLa Hyper cells, which express a fluorescent indicator protein for intracellular ROS. HeLa Hyper cells were treated with or without carotenoids (Car), namely fucoxanthin (Fuco) or neoxanthin (Neo), BL irradiation, or medium change (MC) before BL irradiation. Treatment conditions were as follows: A (Car-, BL-, MC-); B (Car-, BL+, MC-); C (Neo +, BL-, MC-); D (Fuco+, BL-, MC-); E (Neo+, BL+, MC-); F (Fuco+, BL+, MC-); G (Neo+, BL+, MC+); and H (Fuco+, BL+, MC+). In cells treated with fucoxanthin or neoxanthin under BL irradiation, ROS signals were detected regardless of medium change $(\mathrm{E}-\mathrm{H})$. Supplementation with carotenoids alone $(\mathrm{C}, \mathrm{D})$ and $\mathrm{BL}$ irradiation alone (B) did not produce ROS.

Figure 3. ESR spectra of TMPD oxidized by ${ }^{1} \mathrm{O}_{2}$ produced by photo-excited fucoxanthin. 
ESR spectra are shown for samples containing fucoxanthin and TMPD after BL irradiation for (A) $0 \mathrm{~min}$, (B) $20 \mathrm{~min}$, (C) $40 \mathrm{~min}$, (D) $60 \mathrm{~min}$, (E) $80 \mathrm{~min}$ or (F) $100 \mathrm{~min}$. As a control (G), the sample contained histidine in addition to fucoxanthin and TMPD, and was irradiated for 100 min. Spectra were normalized based on the area of Mn markers. The three peaks in the center indicate oxidation of TMPD by ${ }^{1} \mathrm{O}_{2}$.

Figure 4. Peak areas for oxidized TMPD in measured ESR spectra.

Peak areas for oxidized TMPD, as measured in ESR spectra, are indicated; fucoxanthin and TMPD (घ), fucoxanthin and TMPD with hypoxic treatment $(\bullet)$, fucoxanthin, TMPD and histidine $(\boldsymbol{\Delta})$, and $\beta$-carotene and TMPD ( $\square)$. 


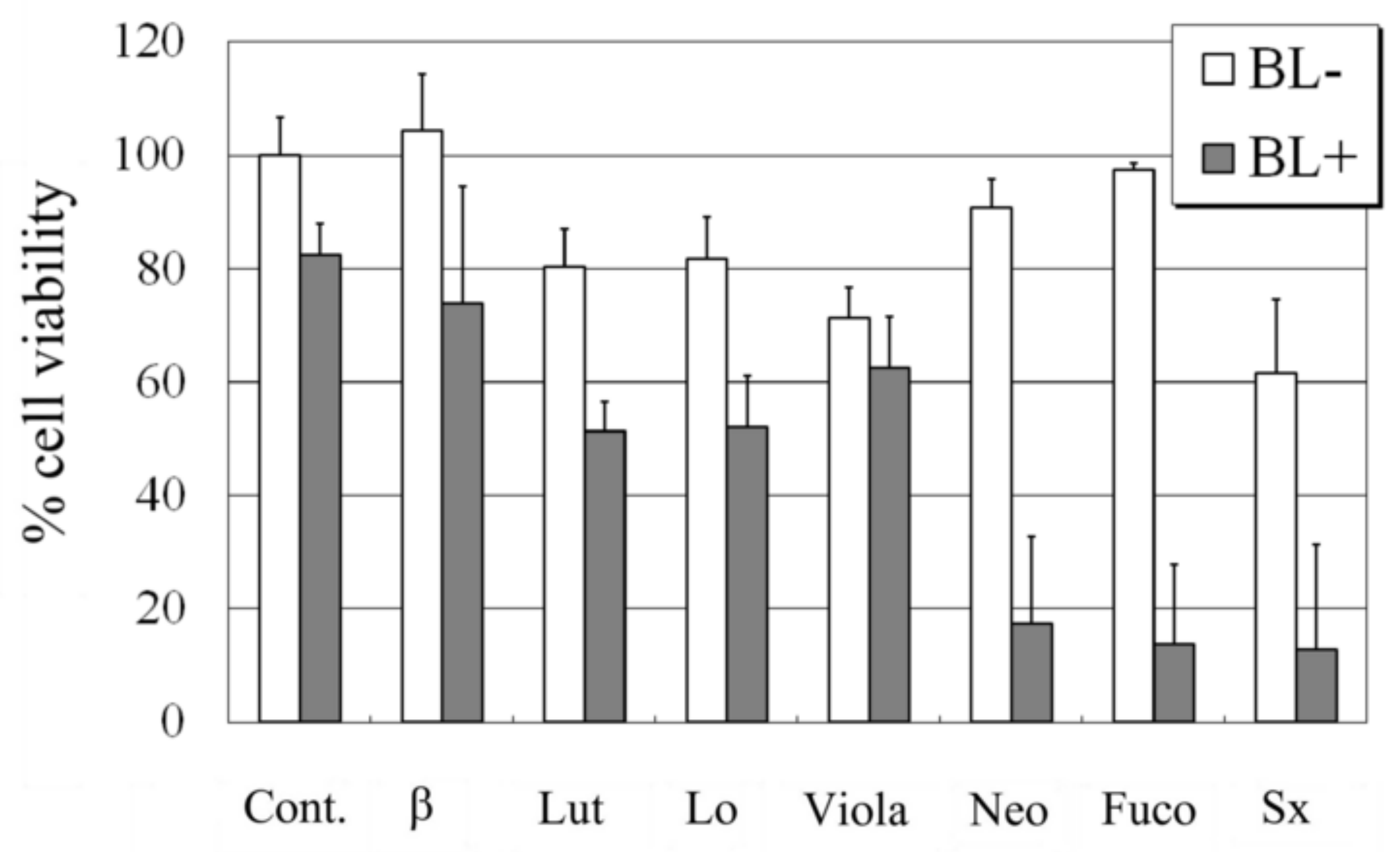

Cont. $\beta$ Lut Lo Viola Neo Fuco Sx 


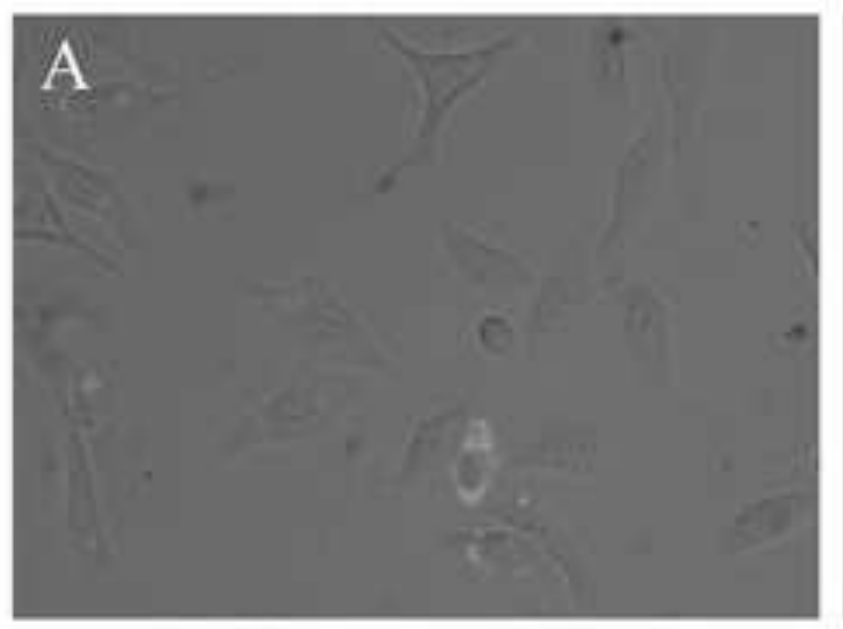

B

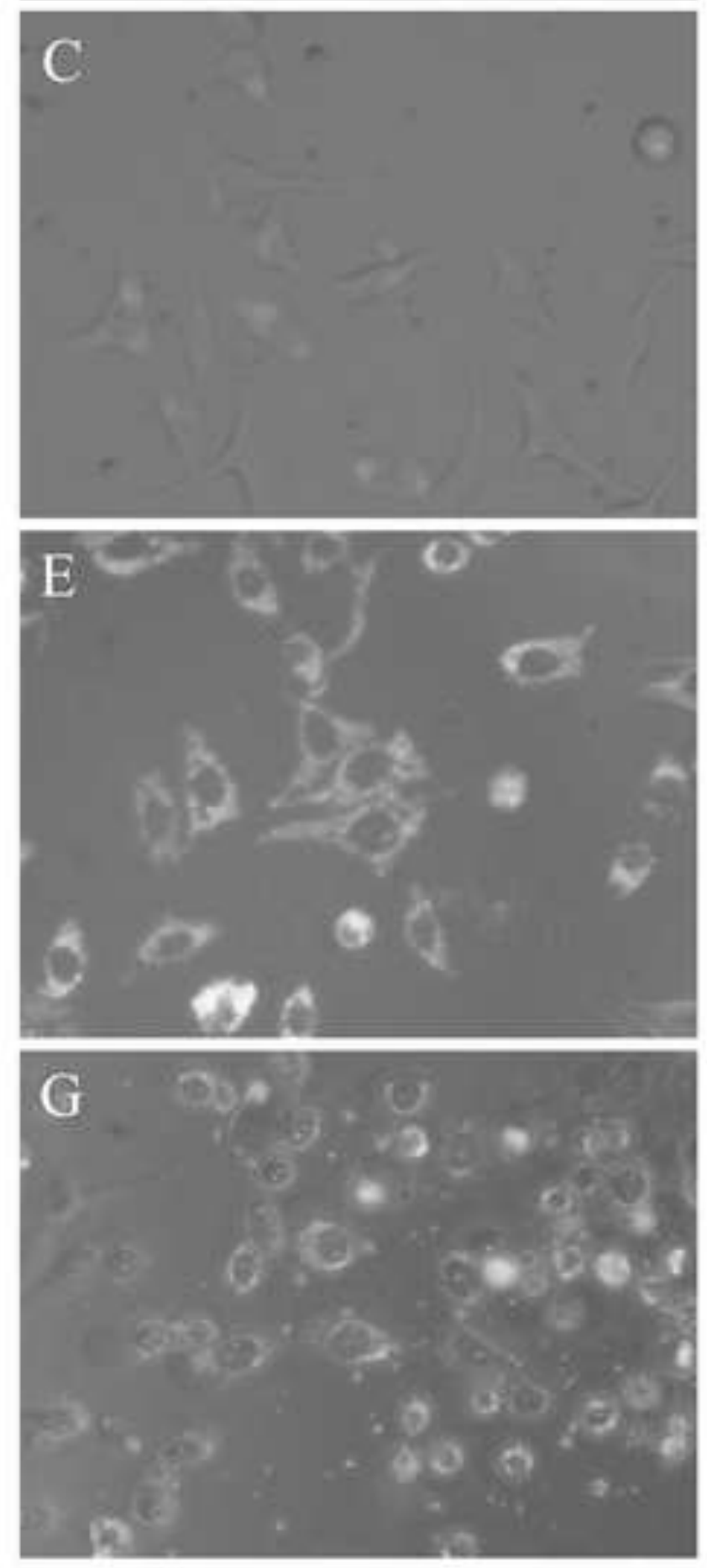

D
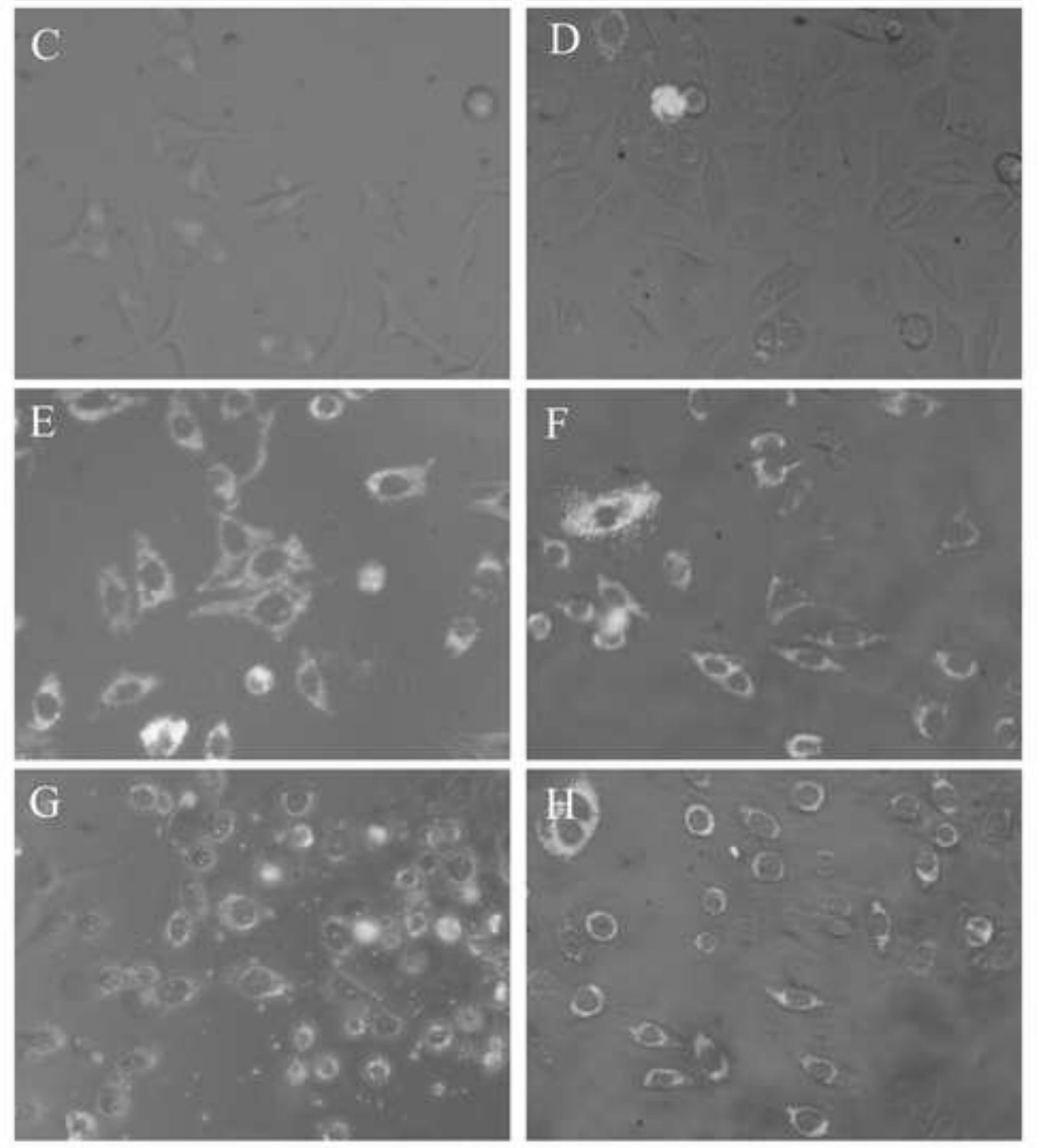


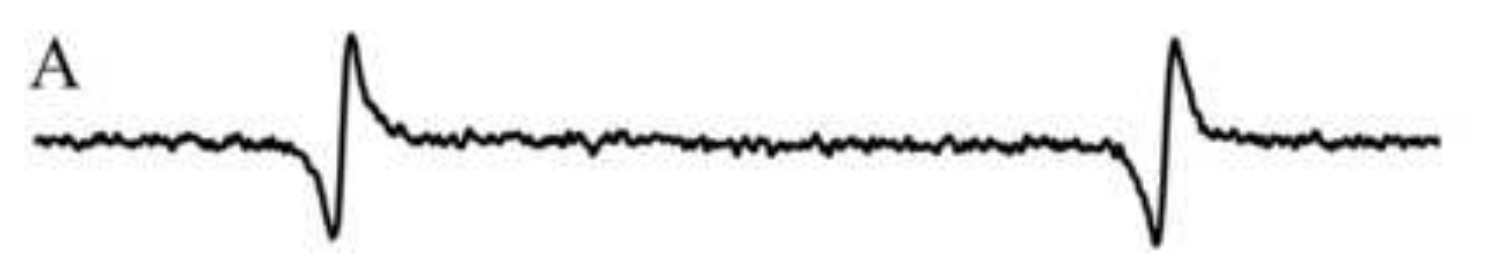

B
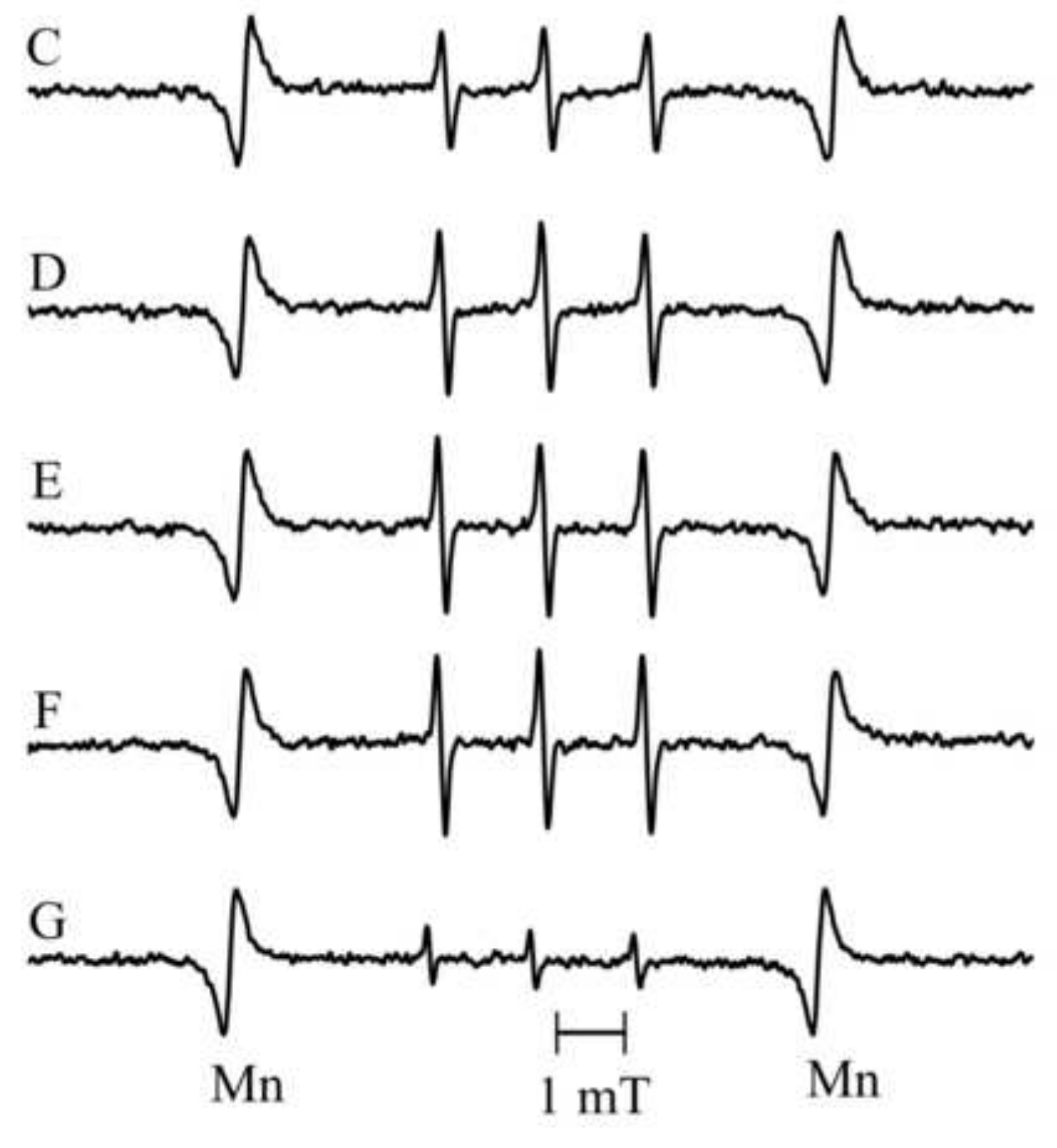

Figure 3

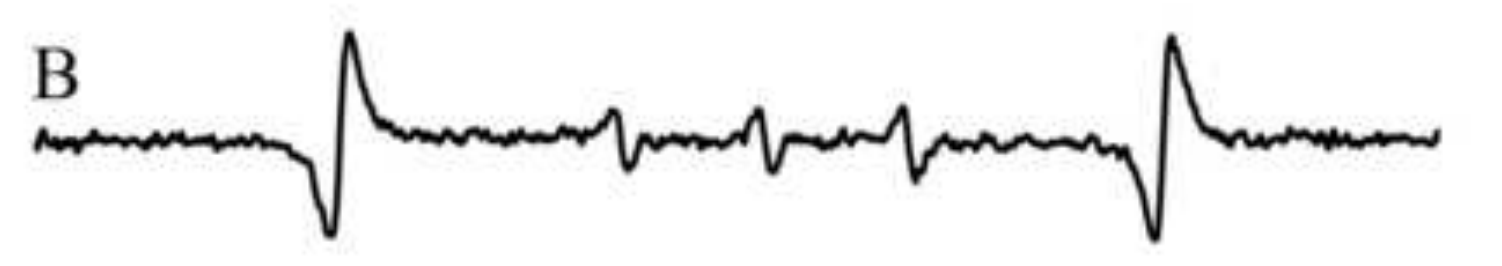

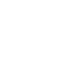
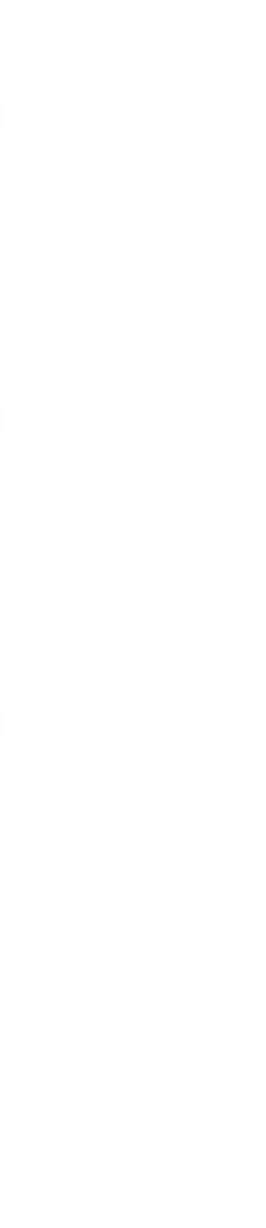

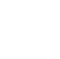

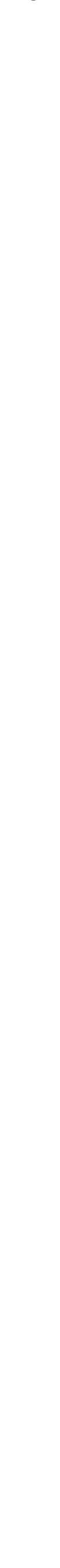


Table 1. Carotenoids used in the present study

\begin{tabular}{lcccc}
\hline Carotenoids & $n^{\mathrm{a}}$ & $\begin{array}{c}\text { Energy of } \mathrm{S}_{1} \text { state } \\
{\left[\mathrm{cm}^{-1}\right]}\end{array}$ & $\begin{array}{c}\text { Energy of } \mathrm{T}_{1} \\
\text { state }^{\mathrm{b}}\left[\mathrm{cm}^{-1}\right]\end{array}$ & $\begin{array}{c}\Delta E^{\mathrm{c}} \\
{\left[\mathrm{cm}^{-1}\right]}\end{array}$ \\
\hline $\begin{array}{l}\beta \text {-Carotene } \\
\text { Lutein } \\
\text { Loroxanthin }\end{array}$ & $9 \beta 2$ & $13900^{\mathrm{d}}$ & 6950 & -932 \\
\hline Violaxanthin & 9 & $14300^{\mathrm{d}}$ & 7150 & -732 \\
\hline Neoxanthin & $8 \mathrm{~A}$ & $14650^{\mathrm{d}}$ & 7325 & -557 \\
\hline $\begin{array}{l}\text { Fucoxanthin } \\
\text { Siphonaxanthin }\end{array}$ & $7 \mathrm{OA}$ & $16520^{\mathrm{e}}$ & 8260 & -332 \\
\hline
\end{tabular}

${ }^{a}$ Number of conjugated $\mathrm{C}=\mathrm{C}$ bonds, according to notation of Polívka and Sundström [10].

${ }^{\mathrm{b}}$ Values were estimated as half of each $\mathrm{S}_{1}$ state energy

${ }^{c}$ Values are energy differences between ${ }^{1} \mathrm{O}_{2}, 7882 \mathrm{~cm}^{-1}$, and $\mathrm{T}_{1}$ state of carotenoids ${ }^{\mathrm{d}}$ Ref. 19

${ }^{\mathrm{e}}$ Ref. 20 